\title{
On a Mathematical Model Describing Optimal Processing Mechanism of Dispersed Granular Materials in Gravitational Flow with Horizontal or Inclined Vibrating Sieve Classifying Screens
}

\author{
Armands Grickus ${ }^{1,2, a}$, Sharif E. Guseynov ${ }^{1,2,3, b}$ \\ ${ }^{I}$ Institute of Fundamental Science and Innovative Technologies, Liepaja University, Liepaja LV-3401, Latvia; \\ ${ }^{2}$ Faculty of Science and Engineering, Liepaja University, 4 Kr.Valdemar Street, Liepaja LV-3401, Latvia; \\ 3"Entelgine" Research \& Advisory Co., Ltd., Kleistu Street 2-53, Riga LV-1067, Latvia; \\ armands.grickus@liepu.lv, ${ }^{b}$ sh.e.guseinov@inbox.lv
}

\begin{abstract}
The investigation of motion and gravitational processing of disperse granular materials is very important for solution of a wide spectrum of technological processes, including the chemical technology of treatment (with or without the decoration-compression procedure) of granular mineral fertilizers and their drying and sorting/separation by means of vibrating sieve classifying screens, in particular. In this work, we have used the apparatus of the theory of continuous media for the mathematical modelling of dynamics of disperse granular materials, and by this we assume that a property of these materials is the distribution of a solid granular component inside of them. The elaborated mathematical model is based on the volume conservation law for granular components, on the momentum conservation law, as well as on the equations for stress tensor in the granular mineral fertilizers and equations for description of the Coulomb granular mineral fertilizers.
\end{abstract}

Keywords: dispersed granular material, gravitational flow, vibrating sieve classifying screen, continuum mechanics, mathematical model.

\section{INTRODUCTION}

The investigation of the motion and mechanics of the gravitational processing of disperse granular materials is important for solution of a wide spectrum of technological processes, including the chemical technology of treatment (with or without the decoration-compression procedure) of granular mineral fertilizers and their drying and sorting/separation by means of vibrating sieve classifying screens, in particular. Apart from technological processes of different kind, the investigation of dynamics of disperse granular media is extremely important for solution of such problems as mineral extraction, engineering, geology - the motion and erosion of soils, in particular, and so on (for instance, see [1]-[7] and the references therein). Currently, there exist two methods for mathematical modelling of the dynamics of disperse granular materials: the first method - this is the consideration of disperse granular media on the level of separate unconnected, but interacting particles ([5], [7]); the second method - this is the consideration of this media from the point of view of continuum mechanics ([3], [4], [6]). When the first method of modelling is used, the flux of finite-size particles is considered, these particles being represented as rigid or elastic geometric figures, e.g., spheres, and it is attempted to derive rules, describing the behaviour of the particle flux as a whole. Significant contributions to the theory of dynamics of disperse granular media have been provided in foreign works (see [8]-[15]) by Reynolds O., Faraday M., Deresiewicz H., Rowe P.W. and Winterkorn H.F., where problems related to artificially excited vibrations, as well as questions of different kind concerning the natural sand flow, have been investigated. Despite of these and other results and their interpretations, which have been obtained by experts in different fields in framework of the first method of modelling, up to now the mechanism of motion of disperse granular media have not provided a satisfactory explanation of many unusual phenomena, arising in motion of such media. When the second method of modelling is used, it is assumed that the properties of particle flux, considered as continuum, can be represented by continuous functions, in such way that any infinitesimal part of disperse granular medium has properties of each separate particle. For example, those mathematical models, which use the apparatus of plasticity theory, are based on criteria of Coulomb-Mohr fluidity; those mathematical models, which use the apparatus of the theory of fluidized boiling beds, are based on thermodynamic principles for particle distribution in bulk, and so on. In this work, the apparatus of http://dx.doi.org/10.17770/etr2017vol3.2547 
continuum mechanics we will use for elaboration of corresponding mathematical models, describing the dynamics of disperse granular materials.

\section{MATHEMATICAL MODELLING}

A. The volume conservation law for granular components and the momentum conservation law

As it was stated in the introduction, we will investigate the dynamics of disperse granular media in gravitational flows from the point of view of continuum mechanics and, hence, we can state that the property of a disperse granular material is the presence of solid granular component, distributed inside of it. Therefore, to take into account the distribution of solid phase in bulk of a continuous granular body (we denote this body by $D_{\text {solid }}$ ), we have to introduce the bulk distribution function $U=U(x, y, z ; t)$, which is a kinematic variable and is continuously defined in the whole region occupied by the body. This function can be interpreted as the porosity, i.e. as the volume fraction of empty spaces (relative free volume), although in reality it is the inverse of this volume fraction. The introduced here function $U(x, y, z ; t)$ can be defined as $U(x, y, z ; t)=1-\Pi(x, y, z ; t)$, where $\Pi(x, y, z ; t)$ denotes the porosity. Obviously, the volume of the granular components in the body $D_{\text {solid }}$ can be defined by the formula

$$
V_{\text {granule }}(t) \stackrel{\text { def }}{\equiv} \int_{D_{\text {solid }}} U(x, y, z ; t) d v
$$

Introducing the notion of mass density $W(x, y, z ; t)$ of granular components, we can determine the total mass $M_{\text {granule of granular }}$ components in the body $D_{\text {solid }}$ by the formula

$$
M_{\text {granule }}(t) \stackrel{\text { def }}{\equiv} \int_{D_{\text {solid }}} U(x, y, z ; t) \cdot W(x, y, z ; t) d v
$$

where $\rho_{\text {v.d. }}(x, y, z ; t) \stackrel{\text { def }}{\equiv} U(x, y, z ; t) \cdot W(x, y, z ; t)$ is the volume density of the investigated disperse granular medium. Further on, since we consider the investigated disperse medium as a continuous media, the mass of the interpore space in the body $D_{\text {solid }}$ is negligibly small. Consequently, the total mass $M_{\text {granule }}(t)$ of the granular components can be considered as the total mass of the granular material and, therefore, the following continuity equation for the considered granular material can obtained from the equation (2):

$$
\frac{\partial \rho_{\mathrm{v} . \mathrm{d} .}(x, y, z ; t)}{\partial t}=-\rho_{\mathrm{v} . \mathrm{d} .}(x, y, z ; t) \operatorname{div}(\vec{\vartheta}(x, y, z ; t))
$$

where $\vec{\vartheta}=\vec{\vartheta}(x, y, z ; t)$ is the spatial vector of the velocity with three Cartesian components $\vartheta_{x}, \vartheta_{y}$, $\vartheta_{z}$; and $\operatorname{div}(\vec{\vartheta}(x, y, z ; t))$ is the divergence of the vector field $\vec{\vartheta}$.

Remark 1. If the granular components (in our case the granular mineral fertilizers) of the body $D_{\text {solid }}$ are incompressible, then mathematically it means that $\frac{\partial W(x, y, z ; t)}{\partial t}=0$ holds. Consequently, in this case the equation

$$
\frac{\partial U(x, y, z ; t)}{\partial t}=-U(x, y, z ; t) \cdot \operatorname{div}(\vec{\vartheta}(x, y, z ; t))
$$

which describes the volume conservation law for the granular components in the body $D_{\text {solid }}$, must be used instead of (3).

It has to be noted that the conservation law (4) can be derived directly from (1). In addition, it is important to notice that, although the volume of granular components is constant, the total volume needs not to be constant, i.e. the total volume can be varied: the positive/negative dilatancy (it is the increase or decrease of the volume at shear deformation) can serve as a natural example in disperse systems of high concentrations, for example, in natural fertilizers. Consequently, in equation (4), as well as in equation (3), $\operatorname{div}(\vec{\vartheta})$ needs not to be zero. Further on, since the total mass of granular mineral fertilizers is calculated from (2), we can write the momentum conservation law in the form of

$$
\rho_{\mathrm{v} . \mathrm{d} .}(x, y, z ; t) \cdot\left(\frac{\partial \vec{\vartheta}(x, y, z ; t)}{\partial t}-\vec{F}\right)=\operatorname{div}(\vec{\sigma}(x, y, z))
$$

or componentwise in the form of

$$
\rho_{\mathrm{v} . \mathrm{d} .}(x, y, z ; t) \cdot\left(\frac{\partial \vartheta_{i}(x, y, z ; t)}{\partial t}-F_{i}\right)=\sigma_{i j, j},
$$

where $\vec{\sigma}$ is the Cauchy stress tensor with nine direct and tangential stresses $\sigma_{x x}=\sigma_{11}, \quad \sigma_{x y}=\sigma_{12}$, $\sigma_{x z}=\sigma_{13}, \sigma_{y x}=\sigma_{21}, \sigma_{y y}=\sigma_{22}, \sigma_{y z}=\sigma_{23}, \sigma_{z x}=\sigma_{31}$, $\sigma_{z y}=\sigma_{32}, \sigma_{z z}=\sigma_{33} ; \vec{F}$ is the vector of mass forces with Cartesian components $F_{x}=F_{1}, F_{y}=F_{2}, F_{z}=F_{3}$; and the covariant derivative is expressed by $\sigma_{i j, j}$. 


\section{B. Equation for the stress tensor in granular mineral fertilizers}

In this subsection, we will consider stresses in the case of non-dissipative granular medium, as well as in the case, where this media is dissipative, i.e. when the energy of ordered macroscopic motions or fields transforms irreversibly into the energy of disordered (i.e. chaotic) motions or fields. Let us assume that specific Helmholtz free energy $A_{\text {H.f.e. }}$ of granular mineral fertilizers depends on the bulk distribution function $U(x, y, z ; t)$ and on its time derivative, as well as on the density of granular components of the body $D_{\text {solid }}$ and temperature $T$, i.e. $A_{\text {H.f.e. }}=A_{\text {H.f.e. }}\left(U, \frac{\partial U}{\partial t}, \rho_{\text {v.d. }}, T\right)$. We can now introduce from thermodynamic considerations the following dynamic quantities:

- pressure $P_{\text {m.p. }}$ of the investigated disperse granular media :

$$
P_{\text {m.p. }} \stackrel{\text { def }}{\equiv} U \cdot W^{2} \cdot \frac{\partial A_{\text {H.f.e. }}}{\partial W}
$$

- spatial pressure $P_{\text {s.p. }}$, which is related to the distribution of granular components in the bulk:

$$
P_{\text {s.p. }} \stackrel{\text { def }}{\equiv} U^{2} \cdot W \cdot \frac{\partial A_{\text {H.f.e. }}}{\partial U} \text {; }
$$

- effort vector $\vec{f}_{\text {e.v. }}$, which is the vector of the balancing stress (as it is related to the system of self-balancing forces, applied either at the pressure centre or shift centre; see e.g. [1]):

$$
\vec{f}_{\text {e.v. }} \stackrel{\text { def }}{\equiv} U \cdot W \cdot \frac{\partial A_{\text {H.f.e. }}}{\partial U} \text {. }
$$

Then, from thermodynamic considerations, we can write

$$
P_{\text {m.p. }} \stackrel{\operatorname{def}}{\equiv} U \cdot \operatorname{div}\left(\vec{f}_{\text {e.v. }}\right)=P_{\text {s.p. }}-P_{\text {m.p. }} .
$$

Equation (9) is a fundamental equation and, being determined by expressions (6)-(8), the dynamical quantities $P_{\text {m.p. }}, P_{\text {s.p. }}, \quad \vec{f}_{\text {e.v. }}$ characterize just the nondissipative part of the stress

$$
\vec{\sigma}^{*} \stackrel{\operatorname{def}}{\equiv} U \cdot \operatorname{div}\left(\vec{f}_{\text {e.v. }}\right)-P_{\text {s.p. }} \cdot \vec{I}-\vec{f}_{\text {e.v. }} \otimes \operatorname{grad}(U),
$$

where the symbol $\otimes$ means the tensor product; the spatial gradient of the scalar function
$U=U(x, y, z ; t)$ is denoted by $\operatorname{grad}(U)$. It follows from the equation (10) and expressions (6)-(8) that the stress is completely determined by Helmholtz energy function in the non-dissipative case: this fact is analogous to the corresponding relation for the equilibrium stress in compressible fluids.

Now, in order to determine the dissipative part of the stress in granular mineral fertilizers, we will use the corresponding equation from the theory of viscous liquid:

$$
\vec{\sigma}-\vec{\sigma}^{*}=v_{1} \operatorname{tr}\left(\vartheta_{\text {s-r.t. }}\right) \vec{I}+2 v_{2} \vartheta_{\text {s-r.t. }},
$$

where $\vartheta_{\text {s-r.t. }}$ is the strain-rate tensor, determined as the symmetrical part of the spatial gradient of velocities; the notion $\operatorname{tr}\left(\vartheta_{\text {s-r.t. }}\right)$ means the trace of the tensor $\vartheta_{\text {s-r.t. }}$ of the second rank (one time covariant rank and one time contravariant rank); quantities $v_{1}=v_{1}(U, W)$ and $v_{2}=v_{2}(U, W)$ are viscosity coefficients.

C. Equation for the description of Coulomb granular mineral fertilizers

The obtained in the previous subsection equation (10) is the general equation for the non-dissipative part of the stress, and just because of this general form it cannot be used for solution of the actual problem: precise expressions are necessary for pressures $P_{\text {m.p. }}$ and $P_{\text {s.p. }}$, as well as for the balancing vector of stresses $\vec{f}_{\text {e.v. }}$. In this section, the required expressions will be obtained. For this purpose, we will assume that specific Helmholtz energy per unit volume $U \cdot W \cdot A_{\text {H.f.e. }}$ is an isotropic function (i.e. it is a tensor function, the symmetry group of which agrees with complete orthogonal group; tensor function - it is a mapping, relating several tensors of different ranks to one tensor of certain rank), which can be expanded in Taylor series in vicinity of $\left.\operatorname{grad}(U)\right|_{U=U_{\text {l.d. }}}=0$, where $U_{\text {l.d. }}$ is the limiting distribution in bulk, which corresponds to the limiting relative fraction of empty spaces in granular mineral fertilizers (the limiting relative fraction of empty spaces in granular mineral fertilizers corresponds to such a state, where volume does not change under shear action; some kinds of granular mineral fertilizers, having larger or smaller relative fraction of empty spaces as compared to its critical value, correspondingly decrease or increase their volume under the shear action, see Remark 1). In addition, we assume that the deviation of $\|\operatorname{grad}(U)\|$ from zero and the deviation of quantity $U$ from $U_{\text {l.d. }}$ is negligibly small. Consequently, within accuracy up to 
terms of the third and higher orders of smallness we can expand the function $U \cdot W \cdot A_{\text {H.f.e. }}$ as follows:

$$
\begin{gathered}
U \cdot W \cdot A_{\text {H.f.e }}=\sum_{i=0}^{2} c_{i} \cdot\left(U-U_{\text {1.d. }}\right)^{i}+ \\
c_{3} \cdot \operatorname{grad}(U) \cdot \operatorname{grad}\left(U_{\text {1.d. }}\right),
\end{gathered}
$$

where $c_{i}=c_{i}\left(U, U_{\text {1.d. }}\right), i=\overline{1,3}$.

In view of the natural requirements of positiveness of the Helmholtz energy $U \cdot W \cdot A_{\text {H.f.e. }}$ per unit volume and of its minimum at the condition $\left.\operatorname{grad}(U)\right|_{U=U_{\text {l.d. }}}=0$, we can write down the following restrictions for the coefficients $c_{i}, i=\overline{0,3}$ :

$$
c_{1}=0, c_{i} \geq 0, i \in\{0,2,3\}
$$

Now, substituting the expansion (12) with coefficients (13) into expressions (6)-(8), we obtain:

$$
\begin{gathered}
P_{\text {m.p. }}=\left(W \cdot \frac{\partial \xi}{\partial W}-\xi\right) \cdot(\operatorname{grad}(U))^{2}+W \cdot \frac{\partial \xi_{0}}{\partial W}-\xi_{0}, \\
P_{\text {s.p. }}=-\eta_{0}+\eta \cdot U^{2}-\xi \cdot(\operatorname{grad}(U))^{2}, \\
\vec{f}_{\text {e.v. }}=2 \cdot \xi \cdot \operatorname{grad}(U),
\end{gathered}
$$

where the following notations are used:

$$
\left.\begin{array}{l}
\xi_{0}=c_{0}+c_{2} \cdot\left(U-U_{\text {l.d. }}\right)^{2}, \\
\eta_{0}=c_{0}+c_{2} \cdot U_{1 . d .}^{2}, \\
\xi=c_{3}, \eta=c_{2} .
\end{array}\right\}
$$

Equation (10) for the stress in non-dissipative situation together with equations (14) and (16) require that non-dissipative direct and tangential stresses, which act on certain area at some point, have a special mutual relation. Note that in the hydrostatics, where the tangential stress must be completely absent, a similar result takes place.

At non-dissipativeness of the considered granular media, the tangential stress has a characteristic nonzero value, which is related to the value of the direct stress. In order to establish this relation mathematically, we will consider an arbitrarily chosen and fixed spatial point and an arbitrary fixed plane with the normal $\vec{n}_{\text {out }}$. Taking into account the equations (10) and (16), the direct stress $\vec{\sigma}$, acting on the fixed plane, is calculated by the following formula:

$$
\vec{\sigma}=\vec{n} \cdot\left(\vec{\sigma}^{*} \cdot \vec{n}\right)=-P_{\text {m.p. }}-2 \cdot \xi \cdot(\operatorname{grad}(U) \cdot \vec{n})
$$

The determined by formula (18) direct stress $\vec{\sigma}$ is related to the tangential stress $\vec{\sigma}_{\text {tang. }}$, acting on the same area, by the following rule:

$$
\begin{aligned}
& \vec{\sigma}^{2}+\vec{\sigma}_{\text {tang. }}^{2}=\left(\vec{\sigma}^{*} \cdot \vec{n}\right)^{2}=P_{\text {m.p. }}^{2}+ \\
& 4 \cdot \xi \cdot P_{\text {m.p. }} \cdot(\operatorname{grad}(U) \cdot \vec{n})^{2}+ \\
& 4 \cdot \xi^{2} \cdot(\operatorname{grad}(U))^{2} \cdot(\operatorname{grad}(U) \cdot \vec{n})^{2}
\end{aligned}
$$

Now, using expressions (18) for exclusion of the term $(\operatorname{grad}(U) \cdot \vec{n})^{2}$ in equation (19) and keeping squares in the final expression, we obtain the following relation:

$$
\vec{\sigma}_{\text {tang. }}^{2}+(\vec{\sigma}-\omega)^{2}=r^{2}
$$

with the introduced here notations

$$
r \stackrel{\text { def }}{\equiv} \xi \cdot(\operatorname{grad}(U))^{2}, \quad \omega \stackrel{\text { def }}{\equiv}-P_{\text {m.p. }}-r
$$

Further on, taking into account equation (14), the second relation in (21) for the pressure $P_{\text {m.p. }}$ takes the following form:

$$
\begin{gathered}
r=\lambda \cdot(\beta-\omega), \\
\frac{1}{\lambda} \stackrel{\text { def }}{\equiv} \frac{W}{\xi} \cdot \frac{\partial \xi}{\partial W}, \quad \beta=\xi_{0}-W \cdot \frac{\partial \xi_{0}}{\partial W}
\end{gathered}
$$

Finally, comparing the expressions (20) and (22), we will find the required relation between the tangential stress $\vec{\sigma}_{\text {tang. }}$ and the direct stress $\vec{\sigma}$, acting on the considered area in the given point of granular mineral fertilizers in the state of non-dissipativeness.

Remark 2. If $\vec{\sigma}$ and $\vec{\sigma}_{\text {tang. }}$ are considered as Cartesian coordinates, then the equation (20) represents a circle with centre at the point $(0, \omega)$ and radius $r$. Besides, the requirement that the radius of this circle must be a function of the shift of its centre from the origin of the coordinate system follows from equation (22), which has been obtained from (20), taking into account (14). If equation (22) is chosen as the necessary and sufficient condition of the nondissipativeness of the media, then this result can be considered as a generalization of the Coulomb condition on stresses at the limiting equilibrium in granular mineral fertilizers, in which the angles of internal friction and bonding are functions of density $W$ and bulk distribution function $U$. Finally, note that in the case if quantities $\lambda$ and $\beta$, determined by formulas (23), are constant, equation (22) becomes an equation of straight line, whereas equation of circle 
(20) transforms into the usual Coulomb-Mohr condition, which is well known as the criterion of fluidity. In this case, the Mohr's circle (20) is tangent to the straight line (22); the scalar functions $\xi_{0}, \xi$,

$\eta_{0}$ and $\eta$, given by formulas (17), can be easily found from the differential equations (23). For example, if $\eta_{0}$ is a constant, then the relation $\eta_{0}=\beta$ follows from the system of equations, consisting of the second formula in (23) and of the first and the third formulas in (17). Its physical interpretation is the following: $\eta_{0}$ corresponds to the bonding of the granular mineral fertilizer and is a strictly positive quantity due to the condition (13) and formulas (17). Thus, if the system of equations, consisting of (10), (11), (15), (16) and the Coulomb condition, is used for representation of the stress tensor in the nondissipative case, then the general determining equation has the form:

$$
\left.\begin{array}{l}
\vartheta_{\text {strain-rate tensor }} \neq 0 \Rightarrow: \\
\vec{\sigma}=v_{1} \cdot \operatorname{tr}\left(\vartheta_{\text {s-r.t. }}\right) \cdot \vec{I}+2 \cdot v_{2} \cdot \vartheta_{\text {s-r.t. }}- \\
2 \cdot \xi \cdot \operatorname{grad}(U) \otimes \operatorname{grad}(U)+\left(\eta_{0}-\eta \cdot U^{2}+\right. \\
\left.\xi \cdot(\operatorname{grad}(U))^{2}+2 \cdot U \cdot \operatorname{div}(\xi \cdot \operatorname{grad}(U))\right) \cdot \vec{I} \\
r=\lambda \cdot(\beta-\omega) .
\end{array}\right\}
$$

The medium, described by equations (24), is called Coulomb granular material.

D. The basic mathematical model of the dynamics of unconnected Coulomb granular materials (with granular mineral fertilizers as an example) with constant density in the regions of dissipative medium.

In this subsection, the dynamics of unconnected Coulomb granular mineral fertilizers is considered with constant density $W(x, y, z ; t) \equiv$ const in the regions, where the medium is not in the state of nondissipativeness. First of all, we note that the nonconnectedness of granular materials (in our case Coulomb granular mineral fertilizers) in mathematical notions means that $\beta=\eta_{0}=0$ holds. Obviously, quantities, characterising the investigated here materials in such regions, are the functions $\xi$ and $\eta$. Consequently, the general determining equation (24), which has been obtained in the previous subsection, takes the following form:

$$
\begin{aligned}
& \vec{\sigma}=v_{1} \cdot \operatorname{tr}\left(\vartheta_{\text {s-r.t. }}\right) \cdot \vec{I}+2 \cdot v_{2} \cdot \vartheta_{\text {s-r.t. }}- \\
& 2 \cdot \xi \cdot \operatorname{grad}(U) \otimes \operatorname{grad}(U)-\left(\eta \cdot U^{2}-\right. \\
& \left.\xi \cdot(\operatorname{grad}(U))^{2}-2 \cdot U \cdot \operatorname{div}(\xi \cdot \operatorname{grad}(U))\right) \cdot \vec{I} ; \\
& r=-\lambda \cdot \omega .
\end{aligned}
$$

The obtained equation (25) is a dynamic relation for the tensor of stresses. The substitution of the equation (25) into the momentum balance equation (5) gives us the following differential equation:

$$
\begin{gathered}
\rho_{\text {v.d. }} \cdot \frac{\partial \vec{\vartheta}}{\partial t}=-\eta \cdot \operatorname{grad}\left(U^{2}\right)+ \\
2 \cdot \xi \cdot U \cdot \operatorname{grad}\left((\operatorname{grad}(U))^{2}\right)+ \\
\left(v_{1}+v_{2}\right) \cdot \operatorname{grad}(\operatorname{div}(\vec{\vartheta}))+ \\
v_{2} \cdot(\operatorname{grad}(U))^{2}+\rho_{\text {v.d. }} \cdot \vec{F}
\end{gathered}
$$

The system of equations, consisting of the equations (4), (5) and (26), describes the cases, where the medium is not in the state of equilibrium, i.e. when it is the dissipative medium. If one analysis the dimensions of differential equations (26) (the equation (26) is a tensorial differential equation and, consequently, it does not consist of a single equation), then it becomes clear that, in addition to the usual dimensionless quantities, appearing in the theory of viscous fluids, it is necessary to consider two extra dimensionless parameters:

- the dimensionless parameter $L$, which is called the relative length and is defined as the characteristic geometric size $\ell$, normalized to the characteristic length of the material, i.e.

$$
L \stackrel{d e f}{\equiv} \ell \cdot \sqrt{\frac{\eta}{\xi}}
$$

- the dimensionless parameter

$$
M \stackrel{\text { def }}{\equiv} \frac{W \cdot g \cdot \ell}{\eta},
$$

where $g$ is gravitational acceleration; the meaning of parameter $M$ - this is the ratio of gravitational forces and forces, responsible for the spatial distribution of granular mineral fertilizers in bulk.

For mathematical completeness and correctness of the elaborated mathematical model (4), (5), (26), it must be completed with the allowed boundary conditions, which ensure the existence (the question of solvability) and uniqueness of the solution. Conditions on the basic variable fields $U$ and $W$, fluxes $\vec{\sigma}$ and $\vec{f}_{\text {e.v. }}$, or on their combination can be used as boundary conditions. For example, the boundary conditions for fluxes

$$
\left.\begin{array}{l}
\vec{\sigma} \cdot \vec{n}=\vec{h} ; \\
\vec{f}_{\text {e.v. }} \cdot \vec{n}=\tau
\end{array}\right\}
$$


can be used, where $\vec{h}$ is the usual effort vector and $\tau$ is the tension, which is related to the vector of stresses. It should be noted here that, since the tension $\tau$ refers to the system of self-balancing forces, the resulting forces are vanishing on the surface and, consequently, there is no need to set $\tau=0$ in the case where free surface is present. However, one should be also noted that the balancing tension is related to the surface energy per unit area, and it influences the effect of surface tension. In the case if both the balancing tension $\tau$ and the effort vector $\vec{h}$ are zero on the boundary, such a boundary is called tensionfree boundary.

\section{NUMERICAL EXPERIMENT}

Carrying out the numerical experiment, a twophase medium of homogeneous solid particles sized $0.5-10 \mathrm{~mm}$ was chosen as the granular medium, in which the granular medium is a dispersed phase, and the dispersion phase is liquid. To simplify the mathematical model (4), (5), (26), (29), the corresponding technological process was considered only on the plane design mesh (see Fig. 1) under the condition that the technological process proceeds at invariable geometry of the inclined vibration classifier, at isothermal conditions and at absence of chemical reactions..

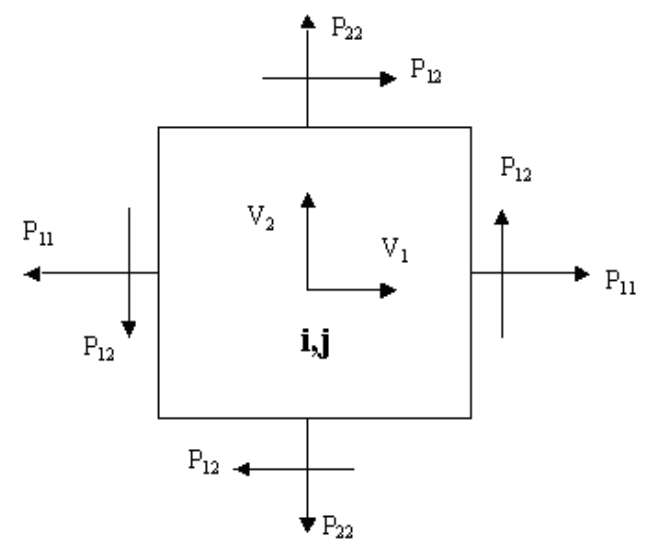

Fig. 1. Plane design mesh for coarse particle of granular dispersion medium.

After taking into account aforesaid suggestions we have got the following simplified mathematical model $(i=1,2 ; j=3-i)$ :

$$
\begin{aligned}
& \frac{\partial \vartheta_{i 1}}{\partial t}+\vartheta_{i 1} \cdot \frac{\partial \vartheta_{i 1}}{\partial x_{1}}+\vartheta_{i 2} \cdot \frac{\partial \vartheta_{i 1}}{\partial x_{2}}=\ddot{\Omega}_{1}-g_{1}+ \\
& \frac{1}{c_{i} \cdot \rho_{0}} \cdot\left(\frac{\partial P_{i 11}}{\partial x_{1}}+\frac{\partial P_{i 12}}{\partial x_{1}}+F_{j i 1}\right),
\end{aligned}
$$

$$
\begin{aligned}
& \frac{\partial \vartheta_{i 2}}{\partial t}+\vartheta_{i 1} \cdot \frac{\partial \vartheta_{i 2}}{\partial x_{1}}+\vartheta_{i 2} \cdot \frac{\partial \vartheta_{i 2}}{\partial x_{2}}=\ddot{\Omega}_{2}-g_{2}+ \\
& \frac{1}{c_{i} \cdot \rho_{0}} \cdot\left(\frac{\partial P_{i 12}}{\partial x_{1}}+\frac{\partial P_{i 22}}{\partial x_{2}}+F_{j i 2}\right), \\
& \frac{\partial c_{i}}{\partial t}+\frac{\partial\left(c_{i} \cdot \vartheta_{i 1}\right)}{\partial x_{1}}+\frac{\partial\left(c_{i} \cdot \vartheta_{i 2}\right)}{\partial x_{2}}=0 \text {, } \\
& P_{111}=\alpha_{0}+\alpha_{0}^{\prime} \cdot\left(\frac{\partial \vartheta_{11}}{\partial x_{1}}+\frac{\partial \vartheta_{12}}{\partial x_{2}}\right)+ \\
& \left(\alpha_{0}^{\prime \prime}+\alpha_{1}^{\prime}\right) \cdot \frac{\partial \vartheta_{11}}{\partial x_{1}} \cdot \frac{\partial \vartheta_{12}}{\partial x_{2}}-\alpha_{0}^{\prime \prime} \cdot\left(\frac{\partial \vartheta_{11}}{\partial x_{2}}+\frac{\partial \vartheta_{12}}{\partial x_{1}}\right)^{2}+ \\
& \alpha_{1} \cdot \frac{\partial \vartheta_{11}}{\partial x_{1}}+\alpha_{1}^{\prime} \cdot\left(\frac{\partial \vartheta_{11}}{\partial x_{1}}\right)^{2}+\alpha_{1}^{\prime \prime} \cdot\left(\frac{\partial \vartheta_{11}}{\partial x_{1}}\right)^{2} \cdot \frac{\partial \vartheta_{12}}{\partial x_{2}}- \\
& \alpha_{1}^{\prime \prime} \cdot \frac{\partial \vartheta_{11}}{\partial x_{1}} \cdot\left(\frac{\partial \vartheta_{11}}{\partial x_{2}}+\frac{\partial \vartheta_{12}}{\partial x_{1}}\right)^{2} \text {, } \\
& P_{112}=\left(\frac{\partial \vartheta_{11}}{\partial x_{1}}+\frac{\partial \vartheta_{12}}{\partial x_{2}}\right) \cdot\left(\alpha_{1}+\alpha_{1}^{\prime} \cdot\left(\frac{\partial \vartheta_{11}}{\partial x_{2}}+\frac{\partial \vartheta_{12}}{\partial x_{1}}\right)\right)- \\
& \alpha_{1}^{\prime} \cdot\left(\frac{\partial \vartheta_{11}}{\partial x_{2}}+\frac{\partial \vartheta_{12}}{\partial x_{1}}\right) \cdot\left(\frac{\partial \vartheta_{11}}{\partial x_{1}}+\frac{\partial \vartheta_{12}}{\partial x_{2}}-\left(\frac{\partial \vartheta_{11}}{\partial x_{2}}+\frac{\partial \vartheta_{12}}{\partial x_{1}}\right)^{2}\right), \\
& P_{122}=\alpha_{0}+\alpha_{0}^{\prime} \cdot\left(\frac{\partial \vartheta_{11}}{\partial x_{1}}+\frac{\partial \vartheta_{12}}{\partial x_{2}}\right)+ \\
& \left(\alpha_{0}^{\prime \prime}+\alpha_{1}^{\prime}\right) \cdot \frac{\partial \vartheta_{11}}{\partial x_{1}} \cdot \frac{\partial \vartheta_{12}}{\partial x_{2}}-\alpha_{0}^{\prime \prime} \cdot\left(\frac{\partial \vartheta_{11}}{\partial x_{2}}+\frac{\partial \vartheta_{12}}{\partial x_{1}}\right)^{2}+ \\
& \alpha_{1} \cdot \frac{\partial \vartheta_{12}}{\partial x_{2}}+\alpha_{1}^{\prime} \cdot\left(\frac{\partial \vartheta_{12}}{\partial x_{2}}\right)^{2}+\alpha_{1}^{\prime \prime} \cdot\left(\frac{\partial \vartheta_{12}}{\partial x_{2}}\right)^{2} \cdot \frac{\partial \vartheta_{11}}{\partial x_{1}}- \\
& \alpha_{1}^{\prime \prime} \cdot \frac{\partial \vartheta_{12}}{\partial x_{2}} \cdot\left(\frac{\partial \vartheta_{11}}{\partial x_{2}}+\frac{\partial \vartheta_{12}}{\partial x_{1}}\right)^{2} \\
& P_{211}=-P+2 \cdot \mu \cdot \frac{\partial \vartheta_{21}}{\partial x_{1}}, \quad P_{212}=\mu \cdot\left(\frac{\partial \vartheta_{22}}{\partial x_{1}}+\frac{\partial \vartheta_{21}}{\partial x_{2}}\right) \text {, } \\
& P_{222}=-P+2 \cdot \mu \cdot \frac{\partial \vartheta_{22}}{\partial x_{2}}, \\
& F_{211}=F_{212}=\left\{\begin{array}{l}
4 \cdot \pi \cdot d^{2} \cdot \rho_{2 i} \cdot \vartheta_{21}^{2} \cdot f_{1} \text { if } c_{\infty} \geq 2 \cdot c_{1}, \\
-\nabla P \cdot c_{1}+\frac{6 \cdot c_{1}}{d} \cdot f_{2} \text { if } c_{\infty}<2 \cdot c_{1},
\end{array}\right. \\
& F_{121}=F_{122}=\pi \cdot d \cdot \vartheta_{12} \cdot\left(0.042 \cdot d \cdot \vartheta_{12} \cdot \rho_{12}+\right. \\
& \left.0.45 \cdot \mu \cdot \sqrt[9]{\left(d \cdot \vartheta_{12} \cdot \rho_{12}\right)^{5}}+3 \cdot \mu\right)
\end{aligned}
$$




$$
\begin{aligned}
& f_{1}=0.042 \cdot d \cdot \vartheta \cdot \rho_{21}+0.45 \cdot \mu \cdot \sqrt[9]{\left(d \cdot \vartheta_{21} \cdot \rho_{21}\right)^{5}}+3 \cdot \mu, \\
& f_{2}=\left(\frac{12 \cdot c_{1}}{1-c_{1}}+3\right) \cdot \frac{\mu \cdot\left(1-2.57 \cdot c_{1}\right)^{2} \cdot\left(\vartheta_{21}-\vartheta_{12}\right)}{d}+ \\
& 0.03 \cdot \rho_{12} \cdot d \cdot \frac{\partial\left(\vartheta_{21}-\vartheta_{12}\right)}{\partial t} .
\end{aligned}
$$

Below there are some graphical results of the numerical implementation of the simplified mathematical model on the application package MathCAD 14.0 (version 14.0.0.163).
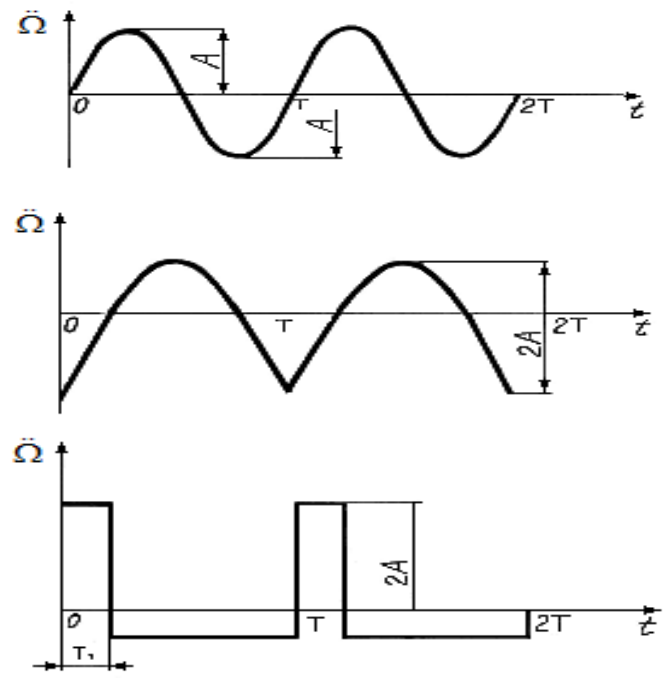

Fig.2. Three different laws of vibration acceleration.

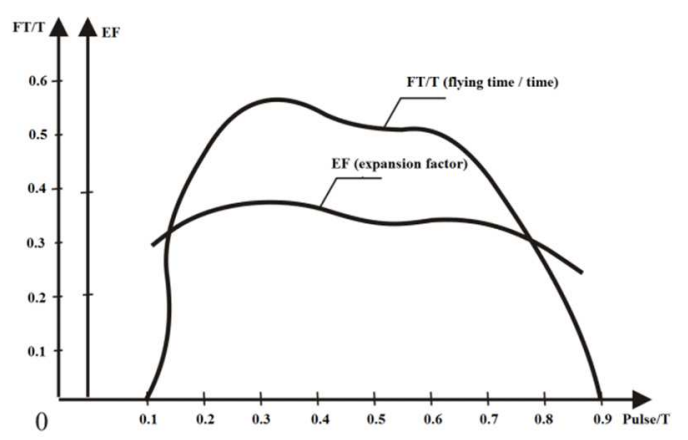

Fig. 3. Dependence of the flight time and the expansion coefficient of granular materials on duration of positive vibration pulse.

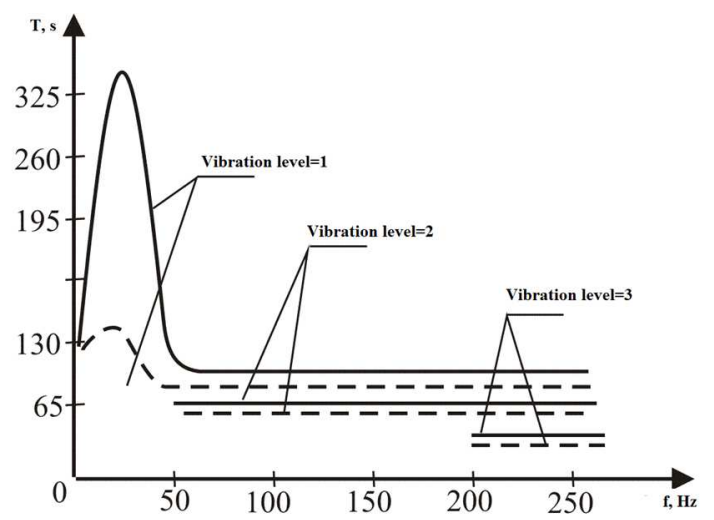

Fig. 4. Dependence of the condensation time of granular structures on the vibration frequency under fixed vibration level $(\mathrm{VL}=\Omega \ddot{g} g)$ : solid line shows harmonic excitation; dashed line shows polyharmonic excitation.
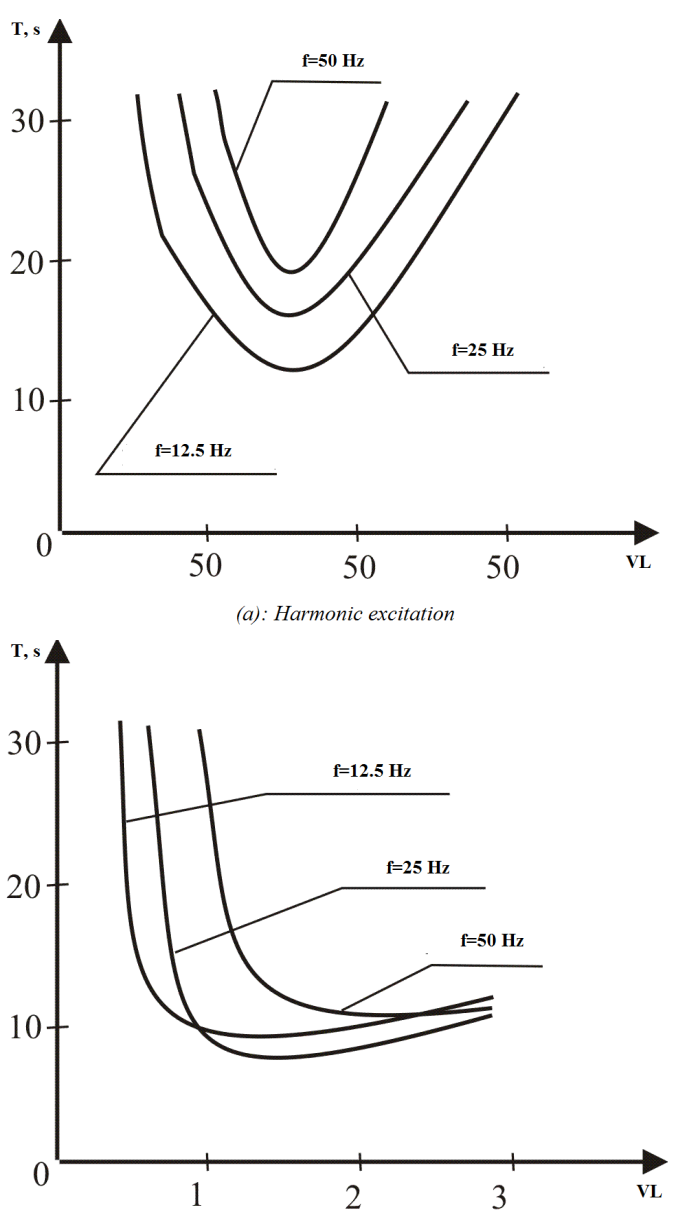

(b): Polyharmonic excitation

Fig. 5. Dependence of the separation time of granular materials on the vibration level $\mathrm{VL}=\Omega \ddot{/} g$ : (a) harmonic excitation; (b) polyharmonic excitation.

\section{IV.CONCLUSION}

In this paper, the mechanism of processing granular disperse materials in gravitational flows, using horizontal or inclined vibrating sieve classifying screens, is investigated. The apparatus of continuum mechanics is chosen as the apparatus of mathematical modelling of the investigated problem and, consequently, we assume that the properties of the particle flow, considered as continuum, can be presented in the form of continuous functions in such a way that each infinitesimal part of the disperse granular medium has characteristic properties of each separate particle.

In the actual paper, based on: (a) volume conservation law of granular components, (b) momentum conservation law, (c) equations, describing Coulombic interactions of granular disperse materials in gravitational flows, (d) equations for the stress tensor in the granular mineral fertilizers (both in the case of non-dissipativeness of granular medium and in the case of its dissipativeness), a mathematical model is built up, which consists of 7 equations (4), (5), (26) and 
boundary conditions (29). In this work, we have analysed also the possibility to consider other boundary conditions, which enable to ensure the existence and uniqueness of the solution for the system of equations (4), (5), (26). The necessity of arising different from (29) boundary conditions is related to the practical point of view: for instance, for a specific type of horizontal (or inclined) vibrating sieve classifying screens, the ensuring of boundary conditions (3) can appear to be unprofitable (economically and/or technically) or even impossible.

It has to be outlined that the elaborated mathematical model describes only the mechanism of motion of granular disperse materials in gravitational fields, but not drying, sorting etc., which are other important problems arising in practice.

\section{ACKNOWLEDGMENTS}

For the second co-author the present article was executed within the framework of The State Research Programme "Next generation Information and Communication Technologies" ("NextIT"), Project No. 4.

\section{REFERENCES}

[1] I. I. Blekhman, Vibration Mechanics. Moscow, Russian Federation: Science, 1994, $374 \mathrm{p}$.

[2] P. V. Klassen, I. G. Grishaev, and I. P. Shomin, Granulation. Moscow, USSR: Chemistry, 1991, $240 \mathrm{p}$.

[3] V. M. Dmitriyev, E. A. Sergeyeva, L. S. Tarova, and S. P. Rudobashta, "The Mathematical Modelling of Mass Transfer in Polydisperse Granular Materials Used in Environment
Protection", Herald of the TSU, vol. 9, No. 4, pp. 456-460, 2004.

[4] S. Savage, "Gravity Flow of Cohesionless Granular Materials in Chutes and Channels", Journal of Fluid Mechanics, vol. 92, No. 1, pp. 53-96, 1979.

[5] P. Evesque, "A Simple Incremental Modelling of GranularMedia Mechanics", Poudres \& Grains, vol. 9, pp.1-12, 1999.

[6] A. L. Svistkov and B. Lauke, "Differential Constitutive Equations of Incompressible Medium at Finite Strains", Applied Mechanics and Theoretical Physics, vol. 50, No. 3, pp. 158-170, 2009.

[7] F. G. Akhmadiev and R. F. Gizzjatov, "Mathematical modelling and optimization of separation processes of disperse materials multistoreyed sith classifier", Proceedings of the Kazan State University of Architecture and Engineering, vol. 18, No. 4, pp. 330-340, 2011.

[8] A. Lyav, Mathematical Theory of Elasticity. Moscow, USSR: ONTI, $1935,647 \mathrm{p}$

[9] H. Deresiewicz, "Mechanics of Granular Matter", Advances in Applied Mechanics, vol. 5, pp. 233-306, 1958.

[10] M. Faraday, "On a Peculiar Class of Acoustical Figures; and on Certain Forms Assumed by Groups of Particles upon Vibrating Elastic Surfaces", Philosophical Trans. Royal Soc. London, vol. 52, pp. 299-340, 1831.

[11] O. Reynolds, "On the Dilatancy of Media Composed of Rigid Particles in Contact", The London, Edinburgh and Dublin Philosophical Magazine and Journal Science, vol. 20, No. 127, pp. 469-483, 1885.

[12] H. M. Jaeger and S. R. Nagel, "Physics of the Granular State", Science, vol. 255, No. 3, pp. 1523-1531, 1995.

[13] P. K. Haff, "Grain Flow as a Fluid-Mechanical Phenomenon", Journal of Fluid Mechanics, vol. 134, pp. 401-430, 1992

[14] P. W. Rowe, The Stress Dilatancy Relation for Static Equilibrium of an Assembly of Particles in Contact", Proceedings of the Royal Society of London, A269, pp. 500$527,1962$.

[15] J. C. Dutertre and H. F. Winterkorn, "Shear Phenomena in Natural Granular Materials", Princeton Soil Engineering Resources, Series 6, Princeton University Press, Princeton, USA, Contract No AFCRRL-66-771, 1966. 